Research Paper

\title{
ADIPOQ rs266729 G/C gene polymorphism and plasmatic adipocytokines connect metabolic syndrome to colorectal cancer
}

\author{
Rosa Divella $^{1 凶}$, Antonella Daniele ${ }^{1}$, Antonio Mazzocca ${ }^{2}$, Ines Abbate ${ }^{1}$, Porzia Casamassima ${ }^{1}$, Cosimo \\ Caliandro3 ${ }^{3}$,Eustachio Ruggeri³ ${ }^{3}$ Emanuele Naglieri ${ }^{4}$, Carlo Sabbà2, Raffaele De Luca ${ }^{3}$ \\ 1. Clinical Pathology Laboratory, Department of Experimantal Oncology. Giovanni Paolo II National Cancer Institute, V.Le Orazio Flacco 65, 70124 -Bari, Italy; \\ 2. Interdisciplinary Department of Medicine, University of Bari School of Medicine, Piazza G. Cesare, 11, 70124 Bari, Italy; \\ 3. Department of Surgery Oncology. Giovanni Paolo II National Cancer Institute, V.Le Orazio Flacco 65, 70124 -Bari, Italy; \\ 4. Unit of Medical Oncology. Giovanni Paolo II National Cancer Institute, V.Le Orazio Flacco 65, 70124 Bari, Italy. \\ $\triangle$ Corresponding author: Rosa Divella, Ph.D., Department of Experimantal Oncology, Clinical Pathology Laboratory National Cancer Institute Giovanni Paolo \\ II, Viale Orazio Flacco, 65, 70100 - Bari, Italy. Tel. +39 805555259; E-mail: rosadive@inwind.it \\ (c) Ivyspring International Publisher. This is an open access article distributed under the terms of the Creative Commons Attribution (CC BY-NC) license \\ (https://creativecommons.org/licenses/by-nc/4.0/). See http://ivyspring.com/terms for full terms and conditions.
}

Received: 2016.09.08; Accepted: 2016.11.27; Published: 2017.03.25

\begin{abstract}
Background: ADIPOQ gene, which encode for Adiponectin (APN), is sited on chromosome 3q27 and linked to a susceptibility locus for metabolic syndrome (MetS). The ADIPOQ rs266729 G/C gene polymorphism is significantly associated with low APN levels and linked to susceptibility to develop cancer. In addition, decreased APN serum levels are linked with tumor development and progression and inversely associated with markers of inflammation. Here, we investigate the influence of APN rs266729 G/C polymorphism on adipocytokine circulating levels and their association with MetS in colorectal cancer patients (CRC).

Methods: Blood samples from 105 CRC patients (50 women and 55 men) with and without MetS were genotyped for APN rs266729 G/C polymorphism by TETRA ARMS PCR. ELISA assay was used to measure plasma levels of APN and inflammatory TNF- $\alpha$ cytokine. Biochemical and anthropometric parameters of MetS were also analyzed.

Results: We found that CRC patients ( $N=75)$ with genotype rs266729G/C or carriers of $\mathrm{G}$ allele were associated with a significantly increased risk of MetS development $(O R=2.9)$ compared to those with CC genotype $(\mathrm{N}=30)$. Also, $C G / G G$ genotypes were associated with significantly lower plasma APN levels and higher TNF- $\alpha$ levels in comparison to $C C$ genotype $(P=0.034)$ and APN levels were decreased in relation to $\mathrm{BMI}$ increases $(\mathrm{P}=0.001)$.

Conclusions: Our findings show that APN rs266729 G/C polymorphism is associated with lower APN levels in CRC patients, indicating that decreased circulating levels of APN may be a determinant risk factor for CRC in MetS patients.
\end{abstract}

Key words: Adiponectin, ADIPOQ gene, TNF-a, colorectal cancer, metabolic syndrome.

\section{Introduction}

Many epidemiological studies suggest an important, but still controversial, role of obesity and adipose tissue (AT) mass in colorectal cancer (CRC) risk and an association with tumor phenotypes [1]. Adipocytes-derived factors, known as adipocytokines, may contribute to the regulation of CRC development and progression [2, 3]. A hallmark of both obesity and cancer is the state of chronic inflammation induced by hypoxia as a consequence of an excessive accumulation of triglycerides within the adipocytes [4, 5]. Under these conditions, AT is altered, resulting in changes in production of steroid hormones and adipokines, metabolic disorders, and chronic subclinical inflammation [6]. These alterations 
have been implicated in carcinogenesis, tumor progression, and metastasis [7]. Adipokines are members of a class of proteins extremely heterogeneous in terms of both structure and function, although they have some common characteristics [8]. From the functional point of view, adipokines modulate the sensitivity of peripheral tissues to insulin, regulate appetite, energy expenditure, glucose and lipids metabolism, homeostasis, angiogenesis, blood pressure and all the axis of endocrine and reproductive systems [9]. Adiponectin (APN) is the product of the ADIPOQ gene sited on chromosome 3q27, which has been linked to a susceptibility locus for metabolic syndrome (MetS), type 2 diabetes and cardiovascular disease [10]. Several studies support the hypothesis that metabolic syndrome or components of metabolic syndrome may be important etiologic factors for certain cancers [11-14]. Epidemiologic data strongly support the observation that people with metabolic syndrome are at increased risk of colorectal adenoma and cancer $[15,16]$. Mortality due to colon cancer and obesity is greatly influenced by both genetic and environmental etiologic factors [17]. Adipokines may play a crucial role in linking these two diseases [18, 19].

Tumor necrosis factor-a (TNF- $a$ ) a pro-inflammatory cytokine is centrally implicated in the pathogenesis of both obesity and cancer [20,21]. TNF-a generates a set of responses or independently or through interaction with other molecules physiologically active. This involves cytotoxic responses to tumors and a consequent increase in the carcinogenesis and metastasis [22, 23]. Elevated plasma and tissue levels of TNF-a observed in obesity leading to the establishment of constant inflammatory state that increases the risk of colon cancer development $[24,25]$. APN is considered to have beneficial antineoplastic effects, which are believed to be due to anti-proliferative, anti-inflammatory effects, along with antagonizing insulin resistance [26]. However, circulating APN level is inversely related with body weight [27]. APN is present in the circulation of healthy humans at high concentrations $[28,29]$. Decreased APN serum levels correlate with tumor development and progression and are inversely associated with markers of inflammation [30]. In particular APN reduces TNF-a induced effects on cell proliferation and migration. In vitro studies indicate that TNF-a, which is elevated in conditions of obesity, may be partially responsible for decreased APN production in obesity [31, 32]. In obesity, decreased APN serum levels correlate with tumor development and progression [33-35]. Therefore, based on this evidence, ADIPOQ gene could be a candidate gene associated with the risk of cancer [36, 37]. APN levels have a strong genetic component. Among the variations of the ADIPOQ gene reported, the SNP rs266729 that is found within the promoter region has been shown to be significantly associated with APN level and is thought to be linked to susceptibility to cancer based on its role in influencing serum APN levels $[38,39]$. APN has been proposed as a determinant factor in the etiology of the MetS, because of its important regulatory action on insulin sensitivity and inflammation [40]. Thus, polymorphism in the ADIPOQ gene may play a role in the pathogenesis of the MetS.

In this study, we evaluated whether ADIPOQ rs266729 G/C gene polymorphism and obesity-related plasmatic adipokines represent a predictive risk factor for colorectal cancer in patients with metabolic syndrome.

\section{Methods}

\section{Patients}

From June 2014 to December 2015, a small sample size of 105 non metastatic CRC patients 50 women and 55 men, median age 70.5 years (33-86) and 50 healthy subjects, median age 56 years were enrolled in this study, at the Giovanni Paolo II National Cancer Institute (NCI) of Bari, Italy. All cases had positive colonoscopic results for malignancy, histologically confirmed as colon cancer. Blood samples were obtained from all subjects at the time of diagnosis. The clinical characteristics of patient (age, sex, therapeutic interventions, etc.) were obtained from medical records. Information regarding weight, height and visceral adiposity of the patients were also recorded. All participants gave written informed consent prior to enrollment in the study, and the Ethical Committee of the NCI approved the protocol in accordance with once the ethical guidelines of the 1975 Declaration of Helsinki.

\section{Anthropometric parameters and blood collection}

Using the height and weight value of all participants, body mass index (BMI) was calculated as weight in kilograms divided by the square of height in meters $(\mathrm{kg} / \mathrm{m} 2)$. Waist circumference was measured with centimeter up to the umbilicus in the standing position after normal expiration. All blood samples of cancer patients were obtained preoperatively. For genotyping whole peripheral blood, $3 \mathrm{ml}$ were collected for all subjects participating in the study. Serum and plasma were collected after a 12-hr fast. For APN and TNF-a ELISA assay, $3 \mathrm{ml}$ of plasma from both patients and healthy donors was 
immediately separated from the cellular fraction by centrifugation at 2,500 r.p.m. $(1,500 \times \mathrm{g})$ for $10 \mathrm{~min}$ and frozen at $-20^{\circ} \mathrm{C}$ until analysis. The analysis of the biochemical profile was conducted in the serum of the subjects immediately after that harvested.

\section{Diagnostic Criteria for Metabolic Syndrome}

The definition of metabolic syndrome varies, however, which may indicate that the associations might be dissimilar. The 3 widely used definitions for metabolic syndrome are: 1) the new International Diabetes Federation (IDF) definition; 2) the National Cholesterol Education Program's Adult Treatment Panel III (ATP III) definition; and 3) the World Health Organization (WHO) clinical criteria for metabolic syndrome [41, 42].

Metabolic syndrome (MetS), is a collection of obesity-associated disorders that comprises dyslipidemia, triglyceride (TG) $>150 \mathrm{mg} / \mathrm{dl}$, high-density lipoprotein (HDL) cholesterol <40 $\mathrm{mg} / \mathrm{dl}$ in males and $<50$ in females, impaired fasting glucose (fasting glucose $\geq 100$ ), visceral adiposity (waist circumference $>102 \mathrm{~cm}$ in men and $>88 \mathrm{~cm}$ in woman) and arterial blood pressure $>130 / 85 \mathrm{mmHg}$. Patients were considered to have MetS when they presented 3 or more of the joint statement criteria from the American Heart Association/National Heart Lung and Blood Institute (AHA/NHLBI) and the International Diabetes Federation (IDF) [43].

\section{Serum biochemical profiles}

Blood fasting serum concentrations of glucose, total cholesterol, low-density lipoprotein (LDL), high-density lipoprotein (HDL) cholesterol and triglycerides were measured using commercially available kits on a Hitachi 7150 analyzer (Hitachi Ltd. Tokyo, Japan).

\section{Genotyping}

Tetra Primer Amplification Refractory Mutation System PCR (tetra ARMS-PCR)

Genotyping was done after extraction of DNA from whole blood using a standard QIAmp kit (QIAGEN Inc.). For detection of adiponectin polymorphism we performed a Tetra amplification refractory mutation system polymerase chain reaction (T-ARMS-PCR) as reported by a previous works [44, 45]. In summary from the National Center for Biotechnology Information (NCBI) we obtained the adiponectin genomic sequence (NT_005612.16). For adiponectin rs266729 polymorphism, we used two external primers (Forward outer: 5`-GGA CTG TGG AGA TGA TAT CTG GGG GGC A-3', Reverse outer: 5 - TGG CCT AGA AGC AGC CTG GAG AAC TGG A-3'), and the two allele specific internal primers were
(Forward inner (C allele): 5`- CTT GCA AGA ACC GGC TCA GAT CCT CCC- 3', Reverse inner (G allele): 5`- GAG CTG TTC TAC TGC TAT TAG CTC TGC-3 $\left.{ }^{\prime}\right)$. The final PCR mixture $(20 \mu \mathrm{l})$ contained DNA $(2 \mu \mathrm{l}), 10 \times$ PCR buffer $(1.5 \mu \mathrm{l}), 2 \mathrm{mM} \mathrm{MgCl} 2$ $\mathrm{MgCl} 2(1.5 \mu \mathrm{l}), 10 \mathrm{mM}$ dNTP $(0.3 \mu \mathrm{l}), 0.25 \mu \mathrm{l}$ of each primer $1 \mathrm{U}$ Taq DNA polymerase and water. The reaction cycle consisted of pre-denaturation at $95{ }^{\circ} \mathrm{C}$ for $2 \mathrm{~min}$, denaturation at $95^{\circ} \mathrm{C}$ for $20 \mathrm{~s}, 35$ cycles of annealing at $56{ }^{\circ} \mathrm{C}$ for $20 \mathrm{~s}$, extension at $72{ }^{\circ} \mathrm{C}$ for $40 \mathrm{~s}$ and a final extension at $72{ }^{\circ} \mathrm{C}$ for $4 \mathrm{~min}$ for complete extension of all PCR fragments. The amplified DNA fragments were verified on a $2 \%$ agarose gel. Each study participant was classified into one of the three possible genotypes: homozygote $\mathrm{C} / \mathrm{C}$, heterozygote C/G or homozygote G/G.

\section{Statistical Analysis}

For the continuous variables, data were analyzed with the Mann-Whitney $U$-test, the unpaired Student's $t$-test and ANOVA. Spearman's correlation was used for the correlation analysis between ADIPOQ and TNF-a. $p$-Values $\leq 0.05$ were considered to be statistically significant. The allelic and genotypic frequencies were estimated by the Chi-square test between patients and controls. Odds ratios (ORs) and $95 \%$ confidence intervals $(95 \% \mathrm{CI})$ were calculated from the logistic model. All statistical analyses were performed by the Number Cruncher Statistical System-Power Analysis and Sample Size Software 2007 (NCSS-PASS, 329 North 1000 East Kaysville, Utah. USA).

\section{Results}

We classified three groups of patients in relation to evaluated BMI $(\mathrm{kg} / \mathrm{m} 2)$, according to the definition of the WHO. Group I including 40 pts normal weight (BMI <25), group II including 34 pts overweight (BMI $\geq 25 \leq 29.9)$ and group III including 31 pts obese with $\mathrm{BMI} \geq 30$.

\section{Adiponectin genotypes and allele frequency in colorectal cancer patients.}

To study the distribution of genotypes and the frequency of alleles of the rs $266729 \mathrm{G} / \mathrm{C}$ genotypes in colorectal cancer patients, we analyzed genomic DNA extracted from whole-blood in EDTA by Tetra Arms PCR analysis. The product sizes for rs266729, 299-bp for control band, 155-bp for C allele, and 201-bp for G allele, are shown in Figure 1 . We found that the frequency of the ADIPOQ G/G genotype $(\mathrm{OR}=3.5$; $p=0.006)$, the presence of the ADIPOQ G allele (G/G and $C / G$ genotypes $)(O R=2.9 ; p=0.02)$ and the frequency of the $A D I P O Q \mathrm{G}$ allele $(\mathrm{OR}=2.8 ; p<0.0001)$ were significantly higher in patients than in controls 
(Table 1) suggesting that this genotype represent a risk factor for development of colorectal cancer. As reported in Table 2, the frequency of the ADIPOQ G allele was significantly higher in patients in overweight $(\mathrm{OR}=5, \mathrm{p}=0.005)$ and in obese $(\mathrm{OR}=4.5$, $\mathrm{p}=0.001)$. Furthermore, the presence of $\mathrm{G}$ allele represents a significant risk factor for developing MetS $(\mathrm{OR}=3.3, \mathrm{p}=0.0004)$ (Table 3).

\section{Association between circulating levels of APN and TNF- $\alpha$ with clinical characteristic in CRC patients}

A significant inverse correlation between the APN and TNF-a circulating levels was found, $p=0,0001$ (Supplementary Information Figure S1). This inverse correlation between these adipokines was found in all the variables considered (Supplementary Information Table S1). In CRC patients, APN plasma levels were significantly lower than controls $(67,3 \pm 45$ vs $135 \pm 30 \mathrm{ng} / \mathrm{ml}$ ), whereas TNF-a plasma levels were much higher than that found in controls (114 \pm 50 vs $6.5 \pm 5,5 \mathrm{pg} / \mathrm{ml}), p<0.0001$. These findings demonstrate that the protective role of APN is nullified by the inflammatory state, in which TNF-a takes part. These data are also corroborated by decreased APN levels $(p<0.0001 ; p=0.01)$ and increased plasma TNF- $\alpha$ $(p=0.0001 ; p=0.03)$ in relation to tumor stage and poorly differentiated tumors. In addition, lower level of APN $(p=0.002)$ and higher level of TNF- $\alpha$ $(p=0.0004)$ were associated to the female gender. Lower APN levels were also associated to rectal cancer $(p=0.01)$. An inverse association was found between plasma APN with BMI $(p=0.0001)$, APN levels decreased in relation to BMI increases whereas plasma TNF- $\alpha$ increases with increasing BMI, $\mathrm{p}=0.0001$, as shown in Figure 2A-B. In our series, a significant decrease in APN $(\mathrm{p}=0.0001)$ and increase of TNF- $\alpha$ circulating levels $(p=0.0001)$ were observed in patients with metabolic syndrome, as shown in Figure 2C-D. Of note, among controls, there was a negative correlation of APN with both BMI and MetS (data not shown). Lowering levels of APN and increased TNF-a suggest that the establishment of inflammatory status predisposes obese patients to MetS.
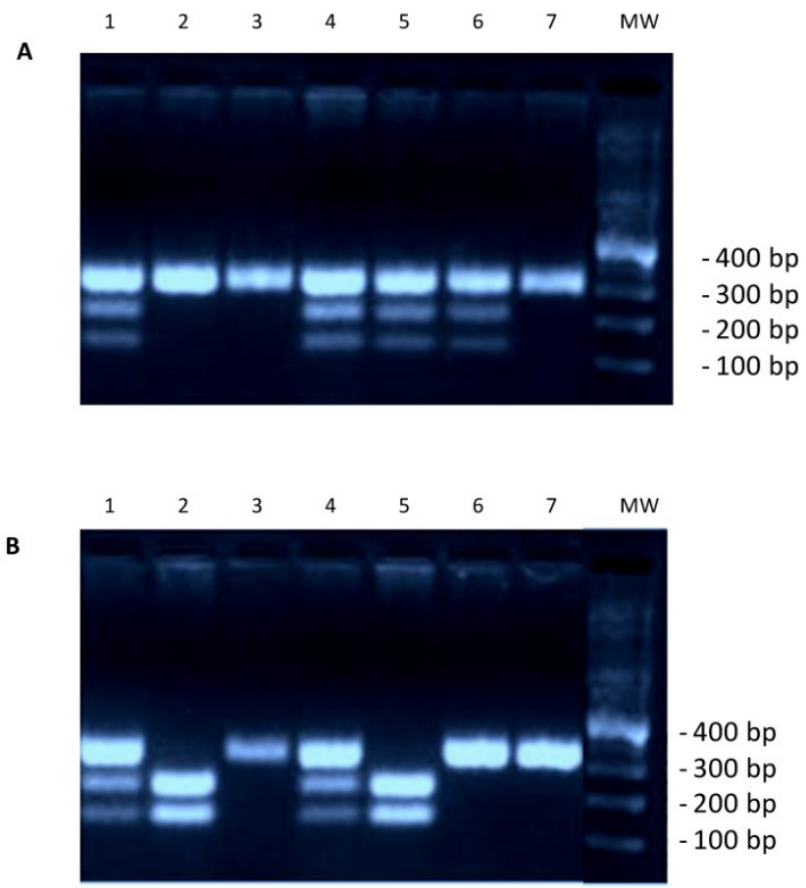

Figure 1. (A) Genotyping result for adiponectin gene rs266729. Marker line 8. CC genotype 334 bp: lines 2, 3, 7. CG genotype 334/212/122 bp: lines 1, 4, 5, 6. (B) Genotyping result for adiponectin gene rs266729. Marker line 8. CC genotype 334 bp: lines 3, 6, 7. CG genotype 334/212/122 bp: lines 1, 4. GG genotype-212/122 bp: Lines $2,5$.

Table 1. ADIPOQ rs $266729 \mathrm{G} / \mathrm{C}$ polymorphism and allelic frequency in patients with colorectal cancer and in controls.

\begin{tabular}{|c|c|c|c|c|c|c|}
\hline & \multicolumn{2}{|c|}{ Patients (N=105) } & \multicolumn{2}{|c|}{ Control (N=50) } & \multirow[t]{2}{*}{ OR (95\% C.I.) } & \multirow[t]{2}{*}{ p-value } \\
\hline & $\mathrm{N}$ & Frequency & $\mathrm{N}$ & Frequency & & \\
\hline \multicolumn{7}{|l|}{ Genotype } \\
\hline $\mathrm{CC}$ & 30 & 0.28 & 27 & 0.54 & reference group & \\
\hline GC & 40 & 0.38 & 14 & 0.28 & $2.5(1.15-5.7)$ & 0.002 \\
\hline GG & 35 & 0.33 & 9 & 0.18 & $3.5(1.4-8.5)$ & 0.006 \\
\hline \multicolumn{7}{|c|}{$\mathrm{X}^{2}=9.75, \mathrm{p}=0.07$} \\
\hline \multicolumn{7}{|c|}{ Genotype } \\
\hline $\mathrm{C} / \mathrm{C}$ & 30 & 0.28 & 27 & 0.54 & reference group & \\
\hline $\mathrm{G} / \mathrm{C}+\mathrm{G} / \mathrm{G}$ & 75 & 0.71 & 23 & 0.46 & $2.9(1.4-5.9)$ & 0.02 \\
\hline \multicolumn{7}{|c|}{$\mathrm{X}^{2}=9.4, \mathrm{p}=0.002$} \\
\hline \multicolumn{7}{|c|}{ Allele } \\
\hline C & 60 & 0.29 & 54 & 0.54 & reference group & \\
\hline G & 145 & 0.71 & 46 & 0.46 & $2.8(1.7-4.6)$ & $<0.0001$ \\
\hline
\end{tabular}


Table 2. - ADIPOQ rs266729 genotypes and allele frequency in relation to BMI, and correlation of ADIPOQ rs266729 genotypes with BMI.

\begin{tabular}{|c|c|c|c|c|c|c|c|c|c|c|}
\hline & \multicolumn{2}{|c|}{ Normal weight $(\mathrm{N}=40)$} & \multicolumn{2}{|c|}{ Overweight $(\mathrm{N}=34)$} & \multicolumn{2}{|c|}{ Obesity $(\mathrm{N}=31)$} & \multirow[t]{2}{*}{ OR (95\% C.I.) vs Over } & \multirow[t]{2}{*}{ p-value } & \multirow[t]{2}{*}{ OR (95\% C.I.) vs Obese } & \multirow[t]{2}{*}{$\mathrm{p}$-value } \\
\hline & $\mathrm{N}$ & frequency & $\mathrm{N}$ & frequency & $\mathrm{N}$ & frequency & & & & \\
\hline \multicolumn{11}{|c|}{ Genotype } \\
\hline $\mathrm{CC}$ & 22 & 0.55 & 4 & 0.11 & 4 & 0.12 & reference group & & reference group & \\
\hline CG & 9 & 0.22 & 20 & 0.58 & 11 & 0.35 & $12.2(3.2-45.9)$ & 0.0002 & $6.7(1.6-26.7)$ & 0.006 \\
\hline GG & 9 & 0.22 & 10 & 0.29 & 16 & 0.51 & $6.1(1.5-24.6)$ & 0.001 & $9.7(2.5-37.4)$ & 0.009 \\
\hline \multicolumn{11}{|c|}{$\mathrm{X}^{2}=26.9, \mathrm{p}<0.0001$} \\
\hline \multicolumn{11}{|c|}{ Genotype } \\
\hline $\mathrm{CC}$ & 22 & 0.22 & 4 & 0.33 & 4 & 0.12 & reference group & & & \\
\hline $\mathrm{CG}+\mathrm{GG}$ & 18 & 0.77 & 30 & 0.66 & 27 & 0.87 & $9.1(2.7-30.8)$ & 0.03 & $8.2(2.4-27.9)$ & 0.0007 \\
\hline \multicolumn{11}{|c|}{$\mathrm{X}^{2}=22.1, \mathrm{p}<0.0001$} \\
\hline \multicolumn{11}{|c|}{ Allele } \\
\hline $\mathrm{C}$ & 24 & 0.40 & 8 & 0.11 & 8 & 0.12 & reference group & & & \\
\hline G & 36 & 0.60 & 60 & 0.88 & 54 & 0.87 & $5(2-12.3)$ & 0.0005 & $4.5(1.8-11.1)$ & 0.001 \\
\hline \multicolumn{11}{|c|}{$X^{2}=18.9, p<0.0001$} \\
\hline
\end{tabular}

Table 3. - ADIPOQ rs266729 genotypes and allele frequency in the MetS and non-Mets groups, and correlation of ADIPOQ rs266729 genotypes with Mets.

\begin{tabular}{|c|c|c|c|c|c|c|}
\hline & \multicolumn{2}{|c|}{ MetS yes $(N=60)$} & \multicolumn{2}{|c|}{ MetS no $(N=45)$} & & \multirow[t]{2}{*}{$p$-value } \\
\hline & $\mathrm{N}$ & Frequency & $\mathrm{N}$ & Frequency & & \\
\hline \multicolumn{7}{|c|}{ Genotype } \\
\hline $\mathrm{CC}$ & 23 & 0.38 & 7 & 0.15 & Reference group & \\
\hline CG & 30 & 0.33 & 20 & 0.44 & $3.2(1.15-9.3)$ & 0.02 \\
\hline GG & 17 & 0.28 & 18 & 0.40 & $3.4(1.2-10)$ & 0.02 \\
\hline \multicolumn{7}{|c|}{$X^{2}=6.5, p=0.03$} \\
\hline \multicolumn{7}{|c|}{ Genotype } \\
\hline $\mathrm{CC}$ & 23 & 0.38 & 7 & 0.15 & Reference group & \\
\hline $\mathrm{CG}+\mathrm{GG}$ & 37 & 0.61 & 38 & 0.84 & $3.37(1.2-8.8)$ & 0.01 \\
\hline \multicolumn{7}{|c|}{$\mathrm{X}^{2}=6.5, \mathrm{p}=0.01$} \\
\hline \multicolumn{7}{|c|}{ Allele } \\
\hline $\mathrm{C}$ & 46 & 0.38 & 14 & 0.15 & Reference group & \\
\hline G & 74 & 0.61 & 76 & 0.84 & $3.3(1.7-6.6)$ & 0.0004 \\
\hline$X^{2}=13, p=$ & & & & & & \\
\hline
\end{tabular}

A

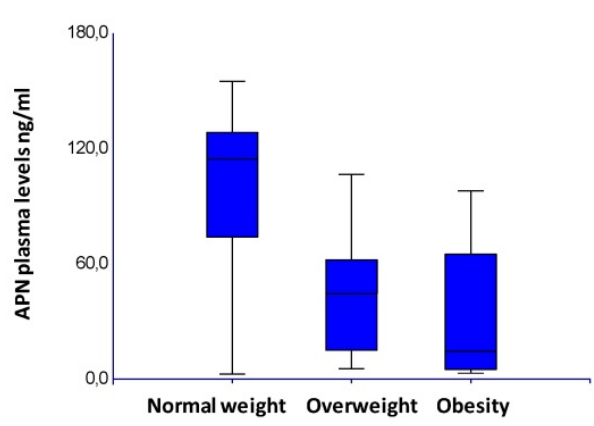

c

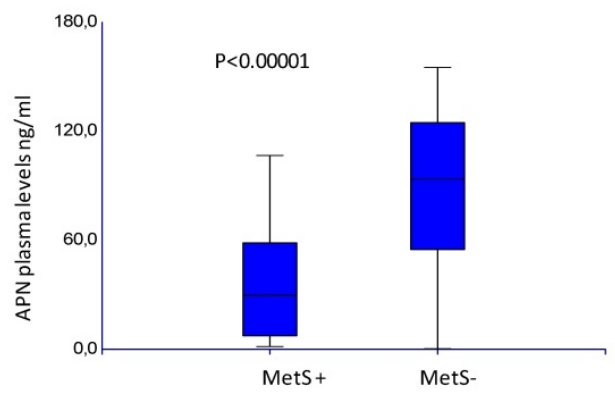

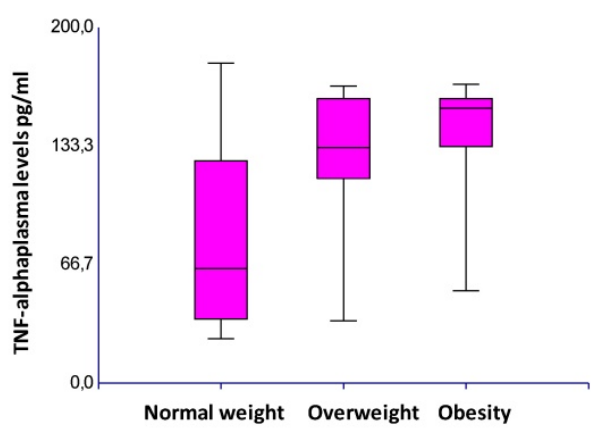

D

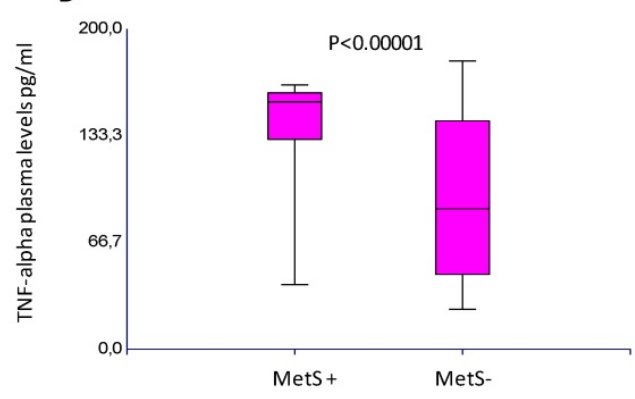

Figure 2. Plasma levels of APN (A) and TNF-alpha (B) in relation to Body Mass Index. Plasma levels of APN (C) and TNF-alpha (D) in relation to Metabolic Syndrome. 
A

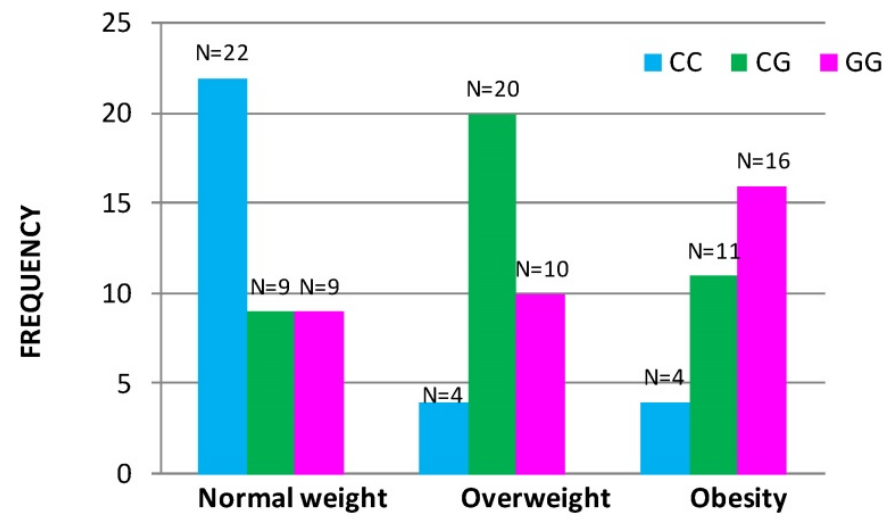

B

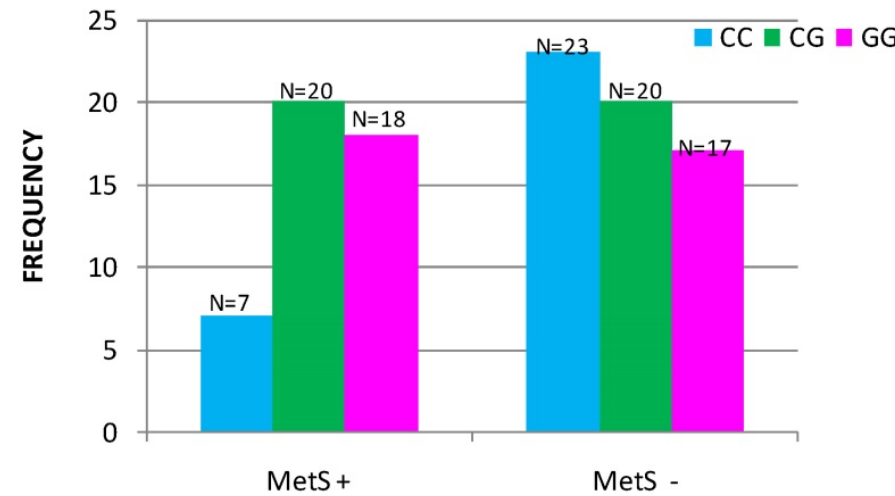

Plasma levels of $A P N$ in accordance to $B M I$

\begin{tabular}{|c|c|c|c|}
\hline Genotype & CC & CG/GG & P-value \\
\hline $\begin{array}{c}\text { Normal } \\
\text { Weight }\end{array}$ & $110 \pm 34$ & $75.7 \pm 38$ & 0.005 \\
\hline $\begin{array}{c}\text { Over } \\
\text { weight }\end{array}$ & $78.8 \pm 30.4$ & $48.2 \pm 32.6$ & 0.08 \\
\hline Obesity & $65.5 \pm 15$ & $43.4 \pm 24.6$ & 0.09 \\
\hline
\end{tabular}

Plasma levels of APN in accordance to MetS

\begin{tabular}{|c|c|c|c|}
\hline Genotype & \multicolumn{1}{|c|}{ CC } & \multicolumn{1}{|c|}{ CG/GG } & P-value \\
\hline MetS+ & $65 \pm 15$ & $39.8 \pm 22.5$ & 0.007 \\
\hline MetS- & $90.9 \pm 43$ & $59 \pm 33$ & 0.005 \\
\hline
\end{tabular}

Figure 3. Distribution of ADIPOQ genotype and plasma levels of APN in accordance to Body Mass Index (A) and to Metabolic Syndrome (B) in patients with colorectal cancer.

\section{Association between rs266729 G/C gene polymorphisms with plasma adiponectin levels in CRC patients correlated to obesity and metabolic syndrome}

We observed that CG/GG genotypes were associated with significantly lower plasma APN levels in comparison to CC genotype $p=0.0001$, ANOVA test Supplementary Information Table S1. Thus, the genotype influences the circulating plasma levels of ADIPOQ in patients with CRC. To assess the discrepancy of plasma APN levels in overweight/obese patients or in the group of patients with MetS, we investigated the association between genetic polymorphism and circulating levels of APN. We found that the frequency of ADIPOQ C/G genotype and $G$ allele were significantly higher in patients with MetS, than in those without MetS and in overweight/obese patients in comparison to patients with normal weight (Figure 3). In particular, we found that 38 CRC patients with genotype rs $266729 \mathrm{G} / \mathrm{C}$ or carriers of $\mathrm{G}$ allele were associated with lower circulating levels of ADIPOQ suggesting a significantly increased risk of MetS development for these patients compared to those with CC genotype $(\mathrm{N}=7)$. In the same way a decrease of circulating APN levels was shown in 57 overweight/obese patients with CG/GG genotype or carriers of $G$ allele compared to patients with normal weight where the higher levels of APN were associated to CC genotype. These data suggest that patients with CC genotype have a lower susceptibility to both obesity and metabolic syndrome compared with patients with CG/GG genotype.

\section{Discussion}

Obesity, a state of low-grade inflammation, is a major health problem associated with an increased risk for MetS and several types of cancer including colorectal cancer. Both obesity and MetS represent important risk factors for development of colorectal cancer [46, 47]. Environmental and metabolic factors interact with genetic predisposition in the pathogenesis of colorectal cancer. The effects of genetic polymorphisms of adiponectin (ADIPOQ) on the risk of cancer incidence have been object of several studies in the last decade [48-50]. APN is linked to central obesity and ADIPOQ variants are promising markers for understanding the genetic base of obesity-related disorders [51]. Our study has identified ADIPOQ rs266729 G/C polymorphism as a 
linker between MetS and inflammation in obese patients as well as a predictive risk factor for developing colorectal cancer in the same patients. In addition, our study demonstrate that plasma APN levels are influenced by the ADIPOQ G/C or G/G genotype which lead to a drastic reduction in circulating levels of APN. APN plasma levels are inversely correlated with increased risk for obesity-related malignancies. In our study, in fact, lower levels of plasma APN were found in overweight, obese and patients suffering with MetS. Weight gain is associated with decreased APN circulating levels, and accumulation of visceral fat may produce inhibiting factors such as TNF-a for APN synthesis or secretion [52]. It is clear from current research that excess visceral adiposity and associated MetS are important predisposition factors for colon rectal cancer development. The evidence that adipocyte hypertrophy and excessive adipose tissue accumulation can promote pathogenic adipocyte and adipose tissue effects, has led to formulate the concept of "adiposopathy", defined as adipocyte and adipose tissue dysfunction. Adiposopathy, together with other factors (TG levels, reduced HDL cholesterol, high blood pressure or impaired glucose metabolism), contributes to the MetS initiation [53]. The causes and the pathophysiological mechanisms that cause inflammatory state associated with obesity are not fully known. However, adipokines may represent the biochemical link between obesity, inflammation and metabolic syndrome. Furthermore, the adiponectin genetic variability strongly determines the full spectrum of this pathological condition that in association with lifestyle (poor eating habits and sedentary lifestyle) predisposes to a greater risk of developing colorectal cancer. In this study, we demonstrated that APN circulating levels are strongly determined by obesity status and that APN expression is inversely correlated with TNF- $\alpha$. The way the adipose tissue expands (increases in size, hypertrophy, and/or in number of cells, hyperplasia) could regulate synthesis and secretion of adiponectin [54]. Drolet et al demonstrated an inverse relationship between mean adipocytes diameter and adiponectin secretion [55]. The hypertrophy of adipose tissue leads to a state of adipocyte hypoxia resulting in the release of inflammatory cytokines and inhibition of adiponectin mRNA [56]. Furthermore, it was shown that the gene promoter activity of APN was down-regulated by hypoxia and TNF-a [57]. The inverse correlation between plasma levels of TNF- $\alpha$ and APN observed in our study confirm the opposite roles exerted by the two cytokines in regulating the inflammatory response and tumor microenvironment.
Alteration of APN and TNF-a signaling in obesity could favor the peritumoral milieu that promotes tumor initiation and progression. We have shown that colorectal cancer patients had a significantly lower APN circulating plasma levels compared with controls and that the G/C genotype and the G allele were associated with a major risk of developing colorectal cancer. We assume that low levels of APN in colorectal cancer are accompanied by reduced adiponectin gene expression in adipose tissue and have been associated with $A D I P O Q$ gene rs266729 $\mathrm{G} / \mathrm{C}$ polymorphism. In our series the inverse correlation between tumor stage and circulating APN suggests that APN may have an important protective role in inflammatory-related carcinogenesis. Our results confirm those shown by Ferroni et al. who demonstrated an inverse correlation between circulating APN levels and tumor stage and were independent predictors of recurrent disease in 60 patients with non-metastatic colorectal cancer [58]. A case-control study consisting of 75 cases of gastric cancer and 52 healthy controls indicated that patients had significantly lower level of plasma ADIPOQ protein compared with controls (mean level, 9.1 vs. 13.3 for cases vs. controls) [59]. The inverse association was also found in colorectal cancer and adenoma. In this study the G allele of rs 266729 G/C were associated with increased risk of colorectal cancer [60]. Our study supports ADIPOQ gene rs266729 G/C polymorphism as a linker between obesity and inflammation and between chronic inflammation and cancer. While research continues to work toward preventing cancers, nutritional and lifestyle modifications may alleviate metabolic syndrome and reduce the risk. In summary, although a prospective study with large sample size is warranted, in these preliminary data, we have demonstrated that rs266729 G/C polymorphism was closely associated with risk of cancer development and that APN and TNF-a exert an opposed impact on the development of colon cancer in obese states. These findings have led to the hypothesis that an unfavorable adipokine profile with a reduction of adipokines with an anti-inflammatory or anti-cancerous activity could serve as a prognostic factor in CRC patients.

\section{Supplementary Material}

Supplementary figure S1 and table S1.

http://www.jcancer.org/v08p1000s1.pdf

\section{Acknowledgments}

The authors wish to thank Eleonora Montenegro, Luisa Cataldo and Domenica Carbonara for their technical support. 


\section{Grants}

This work was supported by grants from Italian Ministry of Health "Ricerca Corrente" - 2013-2015: ADIPOQ SNP rs266729 polymorphism (C-11374G) and expression levels of proinflammatory cytokines involved in the process of carcinogenesis in patients with colorectal cancer: nutritional intervention and proteomic profile in the control of obesity. Grant from Giovanni Paolo II National Cancer Institute, Bari, Italy.

\section{Competing Interests}

\section{interest exists.}

\section{References}

1. Martinez-Useros J, Garcia-Foncillas J. Obesity and colorectal cancer: molecular features of adipose tissue. J Transl Med. 2016; 14(1): 21.

2. Joshi RK, Lee SA. Obesity related adipokines and colorectal cancer: a reviewand meta-analysis. Asian Pac J Cancer Prev. 2014; 15(1): 397-405.

3. Joshi RK, Kim WJ, Lee SA. Association between obesity-related adipokines and colorectal cancer: a case-control study and meta-analysis. World J Gastroenterol. 2014; 20(24): 7941-7949.

4. Matafome P, Santos-Silva D, Sena CM, Seiça R. Common mechanisms of dysfunctional adipose tissue and obesity-related cancers. Diabetes Metab Res Rev. 2013; 29(4): 285-295.

5. Ramos-Nino ME. The role of chronic inflammation in obesity-associated cancers. ISRN Oncol. 2013; 2013:697521.

6. Gilbert CA, Slingerland JM. Cytokines, obesity, and cancer: new insights on mechanisms linking obesity to cancer risk and progression. Annu Rev Med. 2013; 64: 45-57.

7. Nieman KM, Romero IL, Van Houten B, Lengyel E. Adipose tissue and adipocytes support tumorigenesis and metastasis. Biochim Biophys Acta. 2013; 1831(10): 1533-1541.

8. Apostolopoulos V, de Courten MP, Stojanovska L, Blatch GL, Tangalakis K, de Courten B. The complex immunological and inflammatory network of adipose tissue in obesity. Mol Nutr Food Res. 2016; 60(1): 43-57.

9. Schipper, HS, Prakken B, Kalkhoven E, Boes M. Adipose tissue-resident immune cells: key players in immunometabolism. Trends Endocrinol Metabol. 2012; 23: 407-415.

10. Esfahani M, Movahedian A, Baranchi M, Goodarzi MT. Adiponectin: an adipokine with protective features against metabolic syndrome. Iran J Basic Med Sci. 2015; 18(5):430-442.

11. Esposito K, Chiodini P, Colao A, Lenzi A, Giugliano D. Metabolic syndrome and risk of cancer: a systematic review and meta-analysis. Diabetes Care. 2012; 35(11): 2402-2411.

12. Pelucchi C, Serraino D, Negri E, Montella M, Dellanoce C, Talamini R et al. The metabolic syndrome and risk of prostate cancer in Italy. Ann Epidemiol 2011; 21: 835-841.

13. Matthews CE, Sui X, LaMonte MJ, Adams SA, Hébert JR, Blair SN. Metabolic syndrome and risk of death from cancers of the digestive system. Metabolism 2010; 59:1231-1239.

14. Osaki Y, Taniguchi S, Tahara A, Okamoto M, Kishimoto T. Metabolic syndrome and incidence of liver and breast cancers in Japan. Cancer Epidemiol 2012; 36: 141-147

15. Aleksandrova $\mathrm{K}$, Boeing H, Jenab M, Bas Bueno-de-Mesquita $H$, Jansen E, van Duijnhoven FJ, et al. Metabolic syndrome and risks of colon and rectal cancer: the European prospective investigation into cancer and nutrition study. Cancer Prev Res (Phila) 2011; 4: 1873-1883.

16. Shen Z, Wang S, Ye Y, Yin M, Yang X, Jiang $K$ et al. Clinical study on the correlation between metabolic syndrome and colorectal carcinoma. ANZ J Surg 2010; 80: 331-336.

17. Hussan H, Gray DM 2nd, Hinton A, Krishna SG, Conwell DL, Stanich PP. Morbid Obesity is Associated with Increased Mortality, Surgical Complications, and Incremental Health Care Utilization in the Peri-Operative Period of Colorectal Cancer Surgery. World J Surg. 2016; 40(4): 987-994.

18. Surmacz E, Otvos L. Molecular targeting of obesity pathways in cancer. Horm Mol Biol Clin Investig. 2015; 22(2): 53-62.

19. Nimptsch K, Pischon T. Body fatness, related biomarkers and cancer risk: an epidemiological perspective. Horm Mol Biol Clin Investig. 2015; 22(2): 39-51.

20. Muc-Wierzgoń M, Nowakowska-Zajdel E, Dzięgielewska-Gęsiak S, Kokot T, Klakla K, Fatyga E et al. Specific metabolic biomarkers as risk and prognostic factors in colorectal cancer. World J Gastroenterol. 2014; 20(29): 9759-9774.

21. Flores MB, Rocha GZ, Damas-Souza DM, Osório-Costa F, Dias MM, Ropelle $\mathrm{ER}$, et al. Obesity-induced increase in tumor necrosis factor-a leads to development of colon cancer in mice. Gastroenterology 2012; 143(3): 741-53.e1-4.

22. Wang $\mathrm{X}$, Lin $\mathrm{Y}$. Tumor necrosis factor and cancer, buddies or foes? Acta Pharmacologica Sinica 2008; 29(11): 1275- 1288.

23. Li S, Pinard M, Wang Y, Yang L, Lin R, Hiscott J et al. Crosstalk between the TNF and IGF pathways enhances NF- $\mathrm{kB}$ activation and signaling in cancer cells. Growth Horm IGF Res. 2015; 25(5): 253-261.

24. Jain SS, Bird RP. Elevated expression of tumor necrosis factor-alpha signaling molecules in colonic tumors of Zucker obese (fa/fa) rats. Int J Cancer 2010; 127: 2042-2050.

25. Benderska N, Ivanovska J, Rau TT, Schulze-Luehrmann J, Mohan S, Chakilam $\mathrm{S}$ et al. DAPK-HSF1 interaction as a positive-feedback mechanism stimulating TNF-induced apoptosis in colorectal cancer cells. J Cell Sci. 2014; 127(Pt 24): 5273-5287.

26. Hebbard L, Ranscht B. Multifaceted roles of adiponectin in cancer. Best Pract Res Clin Endocrinol Metab. 2014; 28(1): 59-69.

27. Kishida K, Funahashi T, Shimomura I. Adiponectin as a routine clinical biomarker. Best Pract Res Clin Endocrinol Metab. 2014; 28(1):119-130.

28. Lai H, Lin N, Xing Z, Weng H, Zhang H. Association between the level of circulating adiponectin and prediabetes: A meta-analysis. J Diabetes Investig. 2015; 6(4): 416-429.

29. Mather KJ, Goldberg RB. Clinical use of adiponectin as a marker of metabolic dysregulation. Best Pract Res Clin Endocrinol Metab. 2014; 28(1): 107-117.

30. Beg MS, Saleem S, Turer A, Ayers C, de Lemos JA, Khera A et al. A Prospective Analysis of Plasma Adiponectin and Risk of Incident Cancer: The Dallas Heart Study. J Natl Compr Canc Netw. 2015; 13(7): 873-878.

31. Joshi RK, Lee SA. Obesity related adipokines and colorectal cancer: a review and meta-analysis. Asian Pac J Cancer Prev. 2014; 15(1): 397-405.

32. Joshi RK, Kim WJ, Lee SA. Association between obesity-related adipokines and colorectal cancer: a case-control study and meta-analysis. World J Gastroenterol. 2014; 20(24):7941-7949.

33. Dranka-Bojarowska D, Lekstan A, Olakowski M, Jablonska B, Lewinski A, Musialski $P$ et al. The assessment of serum concentration of adiponectin, leptin and serum carbohydrate antigen-19.9 in patients with pancreatic cancer and chronic pancreatitis. J Physiol Pharmacol. 2015; 66(5): 653-563.

34. Ahmed SD, Khanam A, Sultan N, Idrees F, Akhter N. Serum Adiponectin Level Association with Breast Cancer Risk: Evidence from a Case-Control Study. Asian Pac J Cancer Prev. 2015; 16(12): 4945-4948.

35. Divella R, Daniele A, De Luca R, Casamassima P, Savino E, Casamassima A et al. Obesity and colorectal cancer: adiponectin and tumor necrosis factor alfa a representative link between inflammation and cancer. Annals of Oncology 2015; 26 (Supplement 6): vi36-vi52.

36. Park J, Kim I, Jung KJ, Kim S, Jee SH, Yoon SK. Gene-gene interaction analysis identifies a new genetic risk factor for colorectal cancer. J Biomed Sci. 2015; 22: 73.

37. Yang JP, Li X, Wang F, Gao M, Li SL, Chen KS. Association analysis of genetic variants of adiponectin gene and risk of pancreatic cancer. Int J Clin Exp Med. 2015; 8(5):8094-8100.

38. Yang X, Li J, Cai W, Yang Q, Lu Z, Yu J et al. Adiponectin Gene Polymorphisms are Associated with Increased Risk of Colorectal Cancer. Med Sci Monit. 2015; 21: 2595-2606.

39. Yang Y, Zhang F, Ding R, Skrip L, Wang Y, Lei H et al. ADIPOQ gene polymorphisms and cancer risk: a meta-analysis. Cytokine. 2013; 61(2):565-571.

40. Kawada T. Adiponectin and metabolic syndrome: pathway on the relationship. Eur J Clin Nutr 2013; 67: 896.

41. International Diabetes Federation. The IDF Consensus Worldwide Definition of the Metabolic Syndrome. Brussels, Belgium: IDF Communications; 2006.

42. Alberti KG, Zimmet P, Shaw J. Metabolic syndrome-a new world-wide definition. A Consensus Statement from the International Diabetes Federation. Diabet Med. 2006; 23(5): 469-480.

43. Grundy SM, Brewer HB Jr, Cleeman JI, Smith SC Jr, Lenfant C. Definition of metabolic syndrome: report of the National Heart, Lung, and Blood Institute/American Heart Association conference on scientific issues related to definition. Circulation. 2004;109(3): 433-438.

44. Hashemi M, Hanafi Bojd H, Eskandari Nasab E, Bahari A, Hashemzehi NA et al. Association of Adiponectin rs1501299 and rs266729 Gene Polymorphisms With Nonalcoholic Fatty Liver Disease. Hepat Mon. 2013; 13(5): e9527.

45. Medrano RF, de Oliveira CA. Guidelines for the tetra-primer ARMS-PCR technique development. Mol Biotechnol. 2014; 56(7): 599-608.

46. Feakins RM. Obesity and metabolic syndrome: pathological effects on the gastrointestinal tract. Histopathology. 2016; 68(5): 630-640.

47. Trabulo D, Ribeiro S, Martins C, Teixeira C, Cardoso C, Mangualde J et al. Metabolic syndrome and colorectal neoplasms: An ominous association. World J Gastroenterol. 2015; 21(17): 5320-5327.

48. Gu C, Qu Y, Zhang G, Sun L, Zhu Y, Ye D. A single nucleotide polymorphism in ADIPOQ predicts biochemical recurrence after radical prostatectomy in localized prostate cancer. Oncotarget. 2015; 6(31): 32205-32211.

49. Guo X, Liu J, You L, Li G, Huang Y, Li Y. Association between adiponectin polymorphisms and the risk of colorectal cancer. Genet Test Mol Biomarkers. 2015; 19(1): 9-13.

50. Song M, Gong J, Giovannucci EL, Berndt SI, Brenner H, Chang-Claude J et al. Genetic variants of adiponectin and risk of colorectal cancer. Int J Cancer. 2015; 137(1): 154-164. 
51. Narayana Swamy A, Valasala H, Kamma S. In silico Evaluation of Nonsynonymous Single Nucleotide Polymorphisms in the ADIPOQ Gene Associated with Diabetes, Obesity, and Inflammation. Avicenna J Med Biotechnol. 2015; 7(3): 121-127.

52. He $\mathrm{Y}, \mathrm{Lu} \mathrm{L}$, Wei $\mathrm{X}$, Jin D, Qian T, Yu A, et al. The multimerization and secretion of adiponectin are regulated by TNF-alpha. Endocrine. 2016; 51(3): $456-468$.

53. Farb MG, Gokce N. Visceral adiposopathy: a vascular perspective. Horm Mol Biol Clin Investig. 2015; 21(2): 125-136.

54. Wood IS, de Heredia FP, Wang B, Trayhurn P. Cellular hypoxia and adipose tissue dysfunction in obesity. Proc Nutr Soc. 2009; 68(4): 370-377.

55. Drolet R, Belanger C, Fortier M Huot C, Mailloux J, Légaré D et al. Fat depot-specific 'impact of visceral obesity on adipocyte adiponectin release in women. Obesity 2009; 17(3): 424-430.

56. Nagasawa H. Pathophysiological response to hypoxia - from the molecular mechanisms of malady to drug discovery: drug discovery for targeting the tumor microenvironment. J Pharmacol Sci. 2011; 115(4): 446-452.

57. Wensveen FM, Valentić S, Šestan M, Turk Wensveen T, Polić B. The "Big Bang" in obese fat: Events initiating obesity-induced adipose tissue inflammation. Eur J Immunol. 2015; 45(9): 2446-2456.

58. Ferroni P, Palmirotta R, Spila A, Martini F, Raparelli V, Fossile E et al. Prognostic significance of adiponectin levels in non-metastatic colorectal cancer. Anticancer Res 2007; 27: 483-489.

59. Ishikawa M, Kitayama J, Kazama S, Hiramatsu T, Hatano K, Nagawa H. Plasma adiponectin and gastric cancer. Clin Cancer Res. 2005; 11(2 Pt 1): $466-472$.

60. Song M, Gong J, Giovannucci EL, Berndt SI, Brenner H, Chang-Claude J et al. Genetic variants of adiponectin and risk of colorectal cancer. Int J Cancer. 2015; 137(1):154-64. 